\title{
Diffusion Tensor Magnetic Resonance Imaging and Fiber Tractography of the Sacral Plexus in Children with Spina Bifida
}

\author{
Wieke Haakma, Pieter Dik,* Bennie ten Haken, Martijn Froeling, Rutger A. J. Nievelstein, \\ Inge Cuppen, Tom P. V. M. de Jong and Alexander Leemans

\begin{abstract}
Department of Radiology (WH, MF, RAJN) and Image Sciences Institute (AL), University Medical Center Utrecht, Departments of Pediatric Urology (PD, TPVMdJ) and Pediatric Neurology (IC), University Children's Hospitals University Medical Center Utrecht, Utrecht and Neuro Imaging, MIRA Institute for Biomedical Technology and Technical Medicine, University of Twente (BtH), Enschede, The Netherlands, and Department of Forensic Medicine and Comparative Medicine Laboratory, Aarhus University (WH), Aarhus, Denmark
\end{abstract}

Purpose: It is still largely unknown how neural tube defects in spina bifida affect the nerves at the level of the sacral plexus. Visualizing the sacral plexus in 3 dimensions could improve our anatomical understanding of neurological problems in patients with spina bifida. We investigated anatomical and microstructural properties of the sacral plexus of patients with spina bifida using diffusion tensor imaging and fiber tractography.

Materials and Methods: Ten patients 8 to 16 years old with spina bifida underwent diffusion tensor imaging on a 3 Tesla magnetic resonance imaging system. Anatomical 3-dimensional reconstructions were obtained of the sacral plexus of the 10 patients. Fiber tractography was performed with a diffusion magnetic resonance imaging toolbox to determine fractional anisotropy, and mean, axial and radial diffusivity in the sacral plexus of the patients. Results were compared to 10 healthy controls.

Results: Nerves of patients with spina bifida showed asymmetry and disorganization to a large extent compared to those of healthy controls. Especially at the myelomeningocele level it was difficult to find a connection with the cauda equina. Mean, axial and radial diffusivity values at S1-S3 were significantly lower in patients.

Conclusions: To our knowledge this 3 Tesla magnetic resonance imaging study showed for the first time sacral plexus asymmetry and disorganization in 10 patients with spina bifida using diffusion tensor imaging and fiber tractography. The observed difference in diffusion values indicates that these methods may be used to identify nerve abnormalities. We expect that this technique could provide a valuable contribution to better analysis and understanding of the problems of patients with spina bifida in the future.

Key Words: urinary bladder, spinal dysraphism, lumbosacral plexus, diffusion tensor imaging, diagnostic imaging
THE incidence of SB worldwide ranges from 0.3 to $4.5 / 1,000$ births. ${ }^{1,2} \mathrm{~Pa}$ tients with SB generally experience neurogenic bladder dysfunction, and lower limb sensory and motor innervation is affected. ${ }^{3}$ Bladder sphincter contraction and relaxation are coordinated by the central and peripheral nervous systems, and in patients with SB this sphincter function can be affected. ${ }^{4}$ Without treatment the level to which this function is compromised

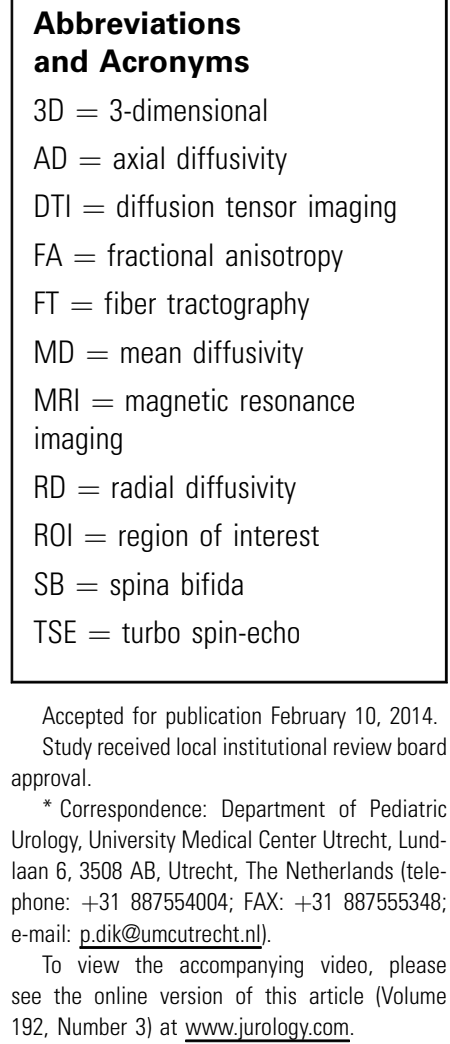

Accepted for publication February 10, 2014

Study received local institutional review board approval.

* Correspondence: Department of Pediatric Urology, University Medical Center Utrecht, Lundlaan 6, 3508 AB, Utrecht, The Netherlands (telephone: +31 887554004; FAX: +31 887555348; e-mail: p.dik@umcutrecht.nl).

To view the accompanying video, please see the online version of this article (Volume 192, Number 3) at www.jurology.com 
determines the prognosis in these patients. Early diagnosis is critical to prevent further neurological impairment but it can be quite arduous due to the complex anatomical configuration and high intersubject variability of peripheral sacral branches. ${ }^{5,6}$ Currently no reliable in vivo, noninvasive routine clinical technique is available to determine how the sacral plexus is organized on an anatomical or structural level in patients with SB.

DTI allows for 3D visualization and structural characterization of nerve tissue. ${ }^{7-9}$ DTI is a MRI technique that is sensitive to the random movement of diffusing water molecules, so-called Brownian motion. Diffusion is more pronounced along the nerves than across their main orientation, causing diffusion to show a high degree of anisotropy in such fibrous tissue. ${ }^{10}$ This anisotropy can be quantified by applying a diffusion-weighted MRI acquisition protocol with multiple diffusion gradient orientations and subsequently estimating the diffusion tensor. $^{7}$ The technique, which enables possible reconstruction of the $3 \mathrm{D}$ architecture of peripheral nerves noninvasively, is referred to as FT. ${ }^{11}$

Although DTI was used in several studies of peripheral nerves, ${ }^{12,13}$ it has been rarely used in the lumbosacral region. ${ }^{5}$ The potential value of DTI to quantify peripheral nerve injury or dysfunction seems promising but the extent to which this can be translated to the clinical setting is still questionable given the numerous fiber constituents that may modulate the observed DTI results. ${ }^{10}$ The 4 diffusion parameters commonly used to investigate tissue microstructural properties are 1) FA, which is high when water molecules move predominantly in 1 direction, 2) MD, which is the average of all eigenvalues (overall amount of diffusion), 3) $\mathrm{AD}$, which is equal to the largest eigenvalue and 4) $\mathrm{RD}$, which is defined as the average of the second and third eigenvalues (fig. 1).

While DTI ${ }^{7,8}$ has been widely used to investigate white matter tracts in the brain, ${ }^{11,14-16}$ its application to peripheral nerves remains limited, mainly due to greater challenges related to data acquisition. ${ }^{5,12,13}$ Takagi et al examined nerve regeneration of the sciatic nerve in rats after contusive injury. ${ }^{17}$ The correlation of FA values with histological and functional changes in that study demonstrated the potential clinical value of DTI for peripheral nerve damage and repair with lower FA values indicating damage to peripheral nerves. Van der Jagt et al recently reported promising progress in reconstructing and analyzing the peripheral sacral nerves using DTI and FT. ${ }^{5}$

We investigated the sacral plexus in 10 children with SB and neurogenic bladder dysfunction using DTI and FT. We compared results to those of healthy controls. We hypothesized that in these
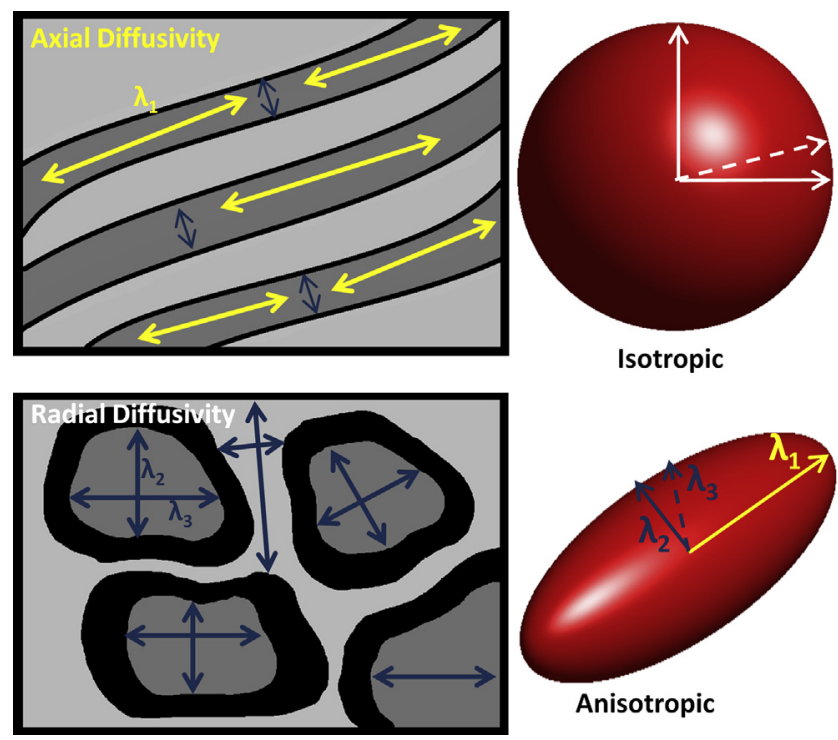

Figure 1. Random movement of water molecules in fluid is isotropic. Diffusion along nerves, represented as AD or $\lambda_{1}$, is higher than perpendicular to nerves, represented as RD or mean of $\lambda_{2}+\lambda_{3}$. Eigenvalues or diffusion lengths $\lambda_{1}, \lambda_{2}$ and $\lambda_{3}$ are represented as being in particular orientation. This diffusion orientation preference is called anisotropy.

patients with SB the microstructural properties of the sacral plexus would show abnormal values of DTI parameters in nerve regions where the tissue structure was affected. Our results revealed that using DTI and FT 1) peripheral sacral nerves in patients with SB and neurogenic bladder dysfunction could be reconstructed and visualized in great detail, 2) the microstructural tissue organization of the sacral plexus could be characterized in vivo and noninvasively, and 3) peripheral nerve tissue abnormalities could be identified at specific locations in the sacral plexus. We believe that investigating nerve tissue properties with DTI and FT, in addition to conventional MRI, would be helpful to better understand the mechanism of the disturbed innervation of the bladder and lower limb muscles in children with SB.

\section{MATERIALS AND METHODS}

\section{Data Acquisition}

Local institutional review board approval was obtained for this study and written informed consent was provided before MRI. Six girls and 4 boys with a mean age of 11.4 years (range 8 to 16 ) with SB and neurogenic bladder dysfunction were included in study. Neural tube defects (myelomeningocele) were mostly located in the lumbar sacral region (L5-S2). To decrease variability in the patient group and keep acquisition time to a minimum MRI was performed from the L4 level to the pelvic floor region. As healthy controls, we used participants in the 
study by van der Jagt et al. ${ }^{5}$ All imaging was done with a 3 Tesla Philips ${ }^{\circledR}$ Achieva MRI System using a 16-channel phased array surface coil. Diffusion-weighted images and anatomical 3D-TSE T2-weighted images were obtained using previously described acquisition protocols. ${ }^{5}$

\section{Data Processing and Analysis}

DTI data sets were processed using the ExploreDTI diffusion MRI toolbox (http://www.ExploreDTI.com). ${ }^{18}$ 1) Data were corrected for subject motion and eddy current induced geometrical distortions. ${ }^{19}$ 2) Diffusion tensors and subsequently diffusion parameters (MD, FA, AD and $\mathrm{RD}$ ) were calculated using the iteratively weighted linear regression procedure. ${ }^{20} 3$ ) A deterministic streamline tractography approach ${ }^{21}$ was used to reconstruct fiber pathways. In each nerve a SEED ROI was placed in the middle of the nerve. A second ROI (AND region) was selected in the nerve root near the cauda equina and another AND ROI was selected more distal along the nerve where it was still traceable. By placing these AND regions according to the 3D-TSE T2-weighted data set fiber we determined trajectories along the entire nerve. Combining a low FA threshold, ie 0.001, with these AND ROIs provides a feasible way to reconstruct tracts with high reproducibility. ${ }^{5}$ Finally, a pediatric neuroradiologist with more than 15 years of experience with MRI evaluated the accuracy of the fiber tract anatomical locations using the anatomical 3D-TSE T2-weighted images.

\section{Statistical Analysis}

$\mathrm{FA}, \mathrm{MD}, \mathrm{AD}$ and $\mathrm{RD}$ values of nerves $\mathrm{L} 4$ to $\mathrm{S} 3$ in patients with SB were investigated and compared with values in healthy controls, as determined in the study by van der Jagt et al. ${ }^{5}$ We investigated variability between the nerves of patients with SB per nerve using the KruskalWallis test. Nerves on the left and right sides were compared using the nonparametric Mann-Whitney U test, which was also used to investigate differences between the nerves of patients with SB and healthy controls. Analysis was done with $\operatorname{SPSS} \AA$, version 21.0.

\section{RESULTS}

With tractography it was possible to obtain $3 \mathrm{D}$ anatomical reconstructions of all 10 patients with SB (supplementary figure, http://jurology.com/). Figure 2 shows FT results of the lumbosacral plexus of a typical patient with SB. The pseudo color encoding represents the magnitude of the diffusion parameters. The table lists diffusion measures of patients with SB and healthy controls. Because MD values appeared to be most indicative of nerve abnormalities (see table), the fiber tracts were color coded for MD (figs. 3 to 5 , see video).

\section{Fiber Tract Evaluation}

The sacral plexus of patients with SB were asymmetrical and disorganized compared to that of healthy controls. In 2 patients nerves at the L5 level could not be reconstructed with FT although they were visible on anatomical T2-weighted images
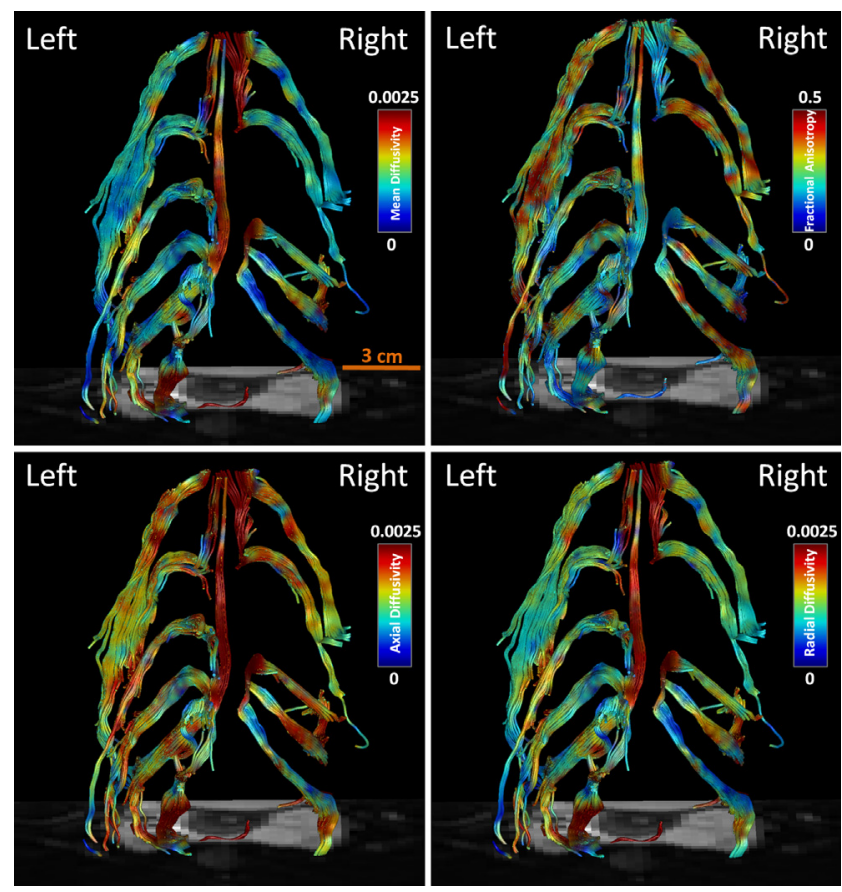

Figure 2. Three-dimensional anatomy of lumbar and sacral nerves at L3-S3 level in typical patient with SB. Color maps show MD, FA, $A D$ and $R D$ along fiber pathways. Red areas represent high diffusivity. Blue areas represent low diffusivity.

(fig. 3, B). S4 and S5 could not be traced in any patient or control. At that level in most cases they were also not visible on anatomical T2-weighted images.

In patients with $\mathrm{SB}$ it was difficult to locate a connection to the cauda equina, especially at the myelomeningocele level (fig. 4, A). In contrast, this connection was clearly visible in healthy controls (fig. 4, B).

\section{Diffusion Parameters}

Statistical testing revealed no significant difference in $\mathrm{FA}, \mathrm{MD}, \mathrm{AD}$ or $\mathrm{RD}$ between the left and right side at the L4-S3 levels ( $p>0.05$ ). Therefore, the 2 sides were pooled for each nerve level. Furthermore, no significant difference was found between individual nerves per level ( $p>0.05)$. The table shows that MD at the L4 level in patients with SB was comparable to that in healthy controls. However, from the L5 level downward significantly lower MD was found in patients than in controls at the S1-S3 levels. Similar differences were noted in $\mathrm{AD}$ and RD.

\section{Bladder Innervation}

In 3 patients we noted a trajectory originating from S2-S4 and continuing to the bladder. Figure 5, A shows an example in 1 patient. Although this could not be confirmed on anatomical T2-weighted images, it is likely that this was the pudendal nerve 
Diffusion parameters of lumbar and sacral nerves of patients with SB and healthy controls at L4-S3 levels

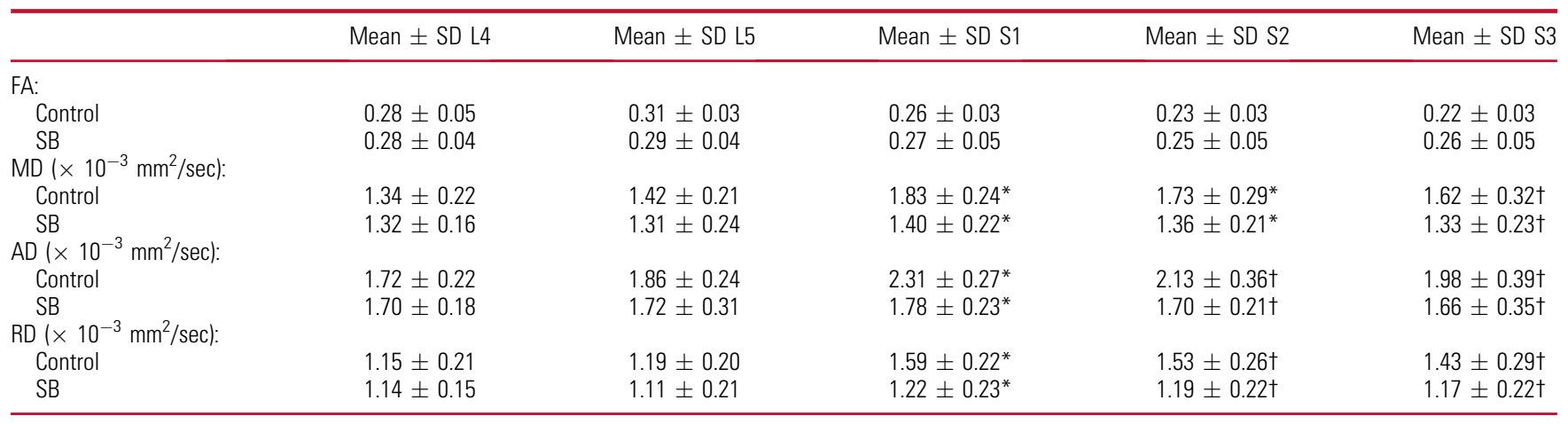

${ }^{*} p<0.001$.

$\dagger p<0.01$.

since it showed a course similar to that on a schematic anatomy configuration of the sacral plexus (fig. 5, B).

\section{DISCUSSION}

To our knowledge we report the first 3 Tesla DTI to reconstruct the $3 \mathrm{D}$ architecture of the sacral plexus in 10 patients with SB that detailed L4-S3 individual pathway trajectories and microstructural properties. Differences in anatomy, particularly asymmetry and disorganization, and MD values in the sacral nerves were found in patients with SB compared to healthy controls.

\section{Interpretation}

Fiber tractography provides striking images of the sacral plexus noninvasively. ${ }^{5}$ However, this technology has not been extensively used to investigate peripheral nerves since DTI and FT are not straightforward or trivial to apply. In addition, interpretation is a major difficulty of DTI. ${ }^{22} \mathrm{Ab}$ normalities in fiber reconstructions and diffusion parameters can also be caused by deformations and artifacts in data or limitations of the imaging technique. $^{23,24}$

\section{Clinical Relevance}

In this study MD values from L5 to the caudal level were lower in patients with SB than controls but for L4, which was still intact, MD values were similar in the groups. The decrease in MD could involve reduced intrinsic diffusion in the intra-axonal space due to cytoskeletal breakdown, resulting in increased viscosity. ${ }^{10}$ It could also be related to myelomeningocele, which in these patients was mostly located at the L5-S1 level. At that level it was also difficult to find a connection to the cauda equina. With some abuse of terminology, if nerves at that level do not work properly, they
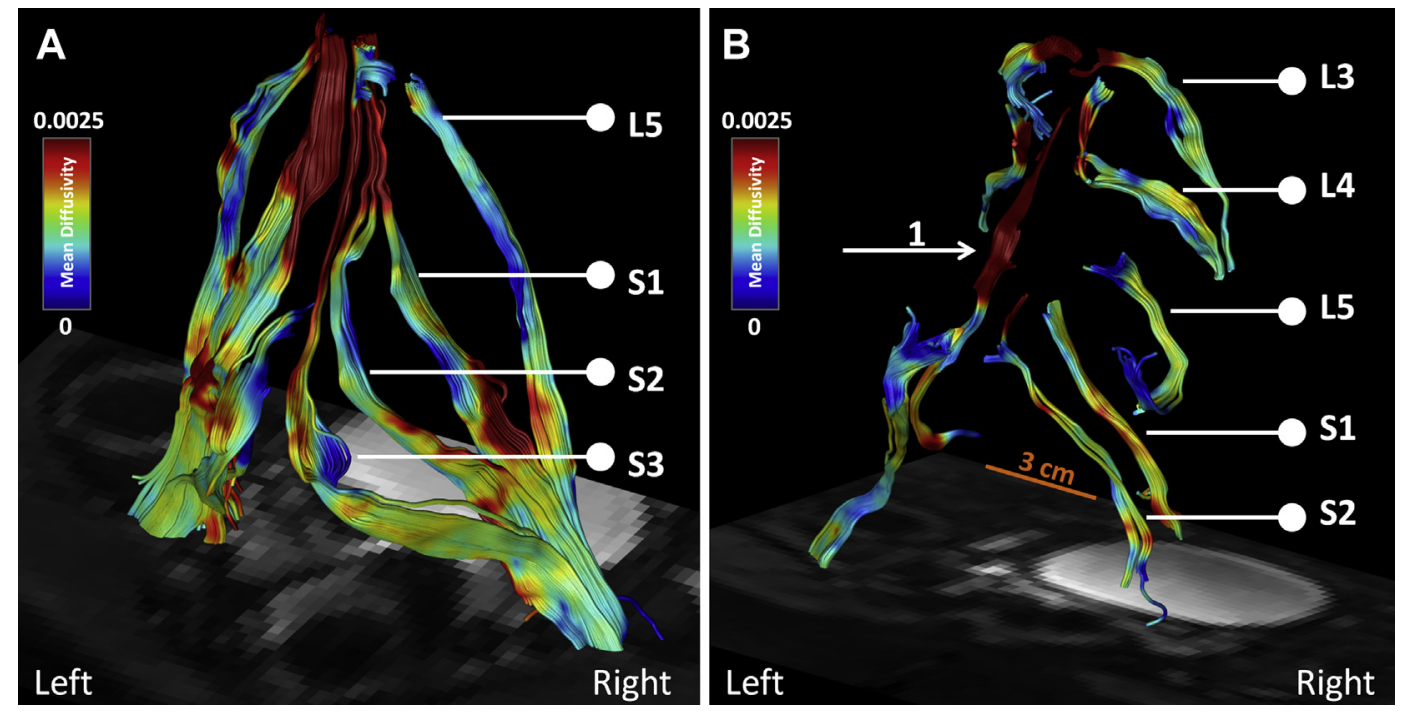

Figure 3. Lower lumbar and sacral nerves. $A$, healthy control. $B$, patients with SB and myelomeningocele from L5 level to caudal. On left side L5 (1) could not be reconstructed. 

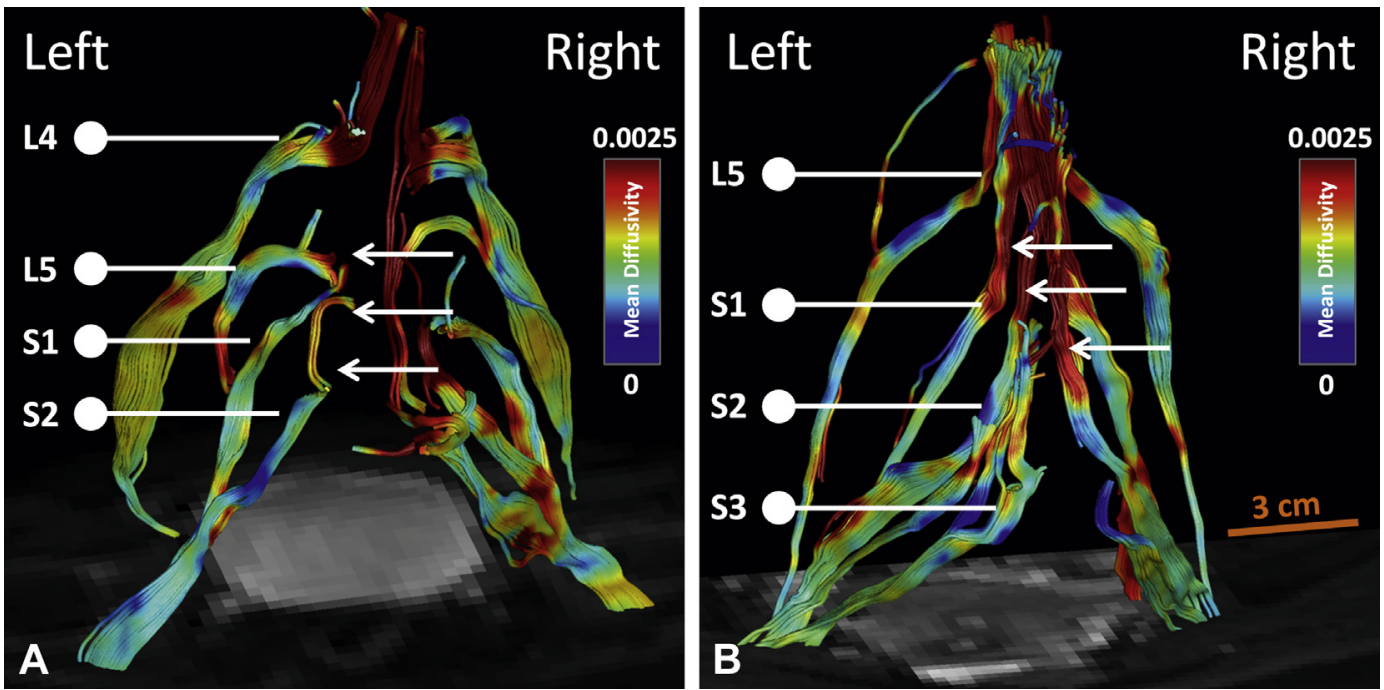

Figure 4. Posterior view. $A$, patient with SB and myelomeningocele at L5-S1 lumbosacral level. Sacral nerves do not connect (arrows) with cauda equina. $B$, in healthy control nerves connect to cauda equina.

might be compared to an electric cable that is not adequately plugged into the socket. To investigate whether the lower MD values of these nerves were caused by myelomeningocele, results could be compared to those in patients with SB in whom myelomeningocele is located at a higher level (thoracic or high lumbar).

DTI revealed a trajectory to the bladder that was likely the pudendal nerve. The anatomical image did not show this trajectory. Because the pudendal nerve originates from $\mathrm{S} 2-\mathrm{S} 4$, it would be expected that the pudendal nerve would also have lower MD values. Future studies of the correlation between urodynamic findings and DTI metrics in the sacral plexus of patients with SB may support this hypothesis. The patient group could be extended by investigating other sacral malformations such as anorectal malformation. ${ }^{25}$ Lower lesions are often
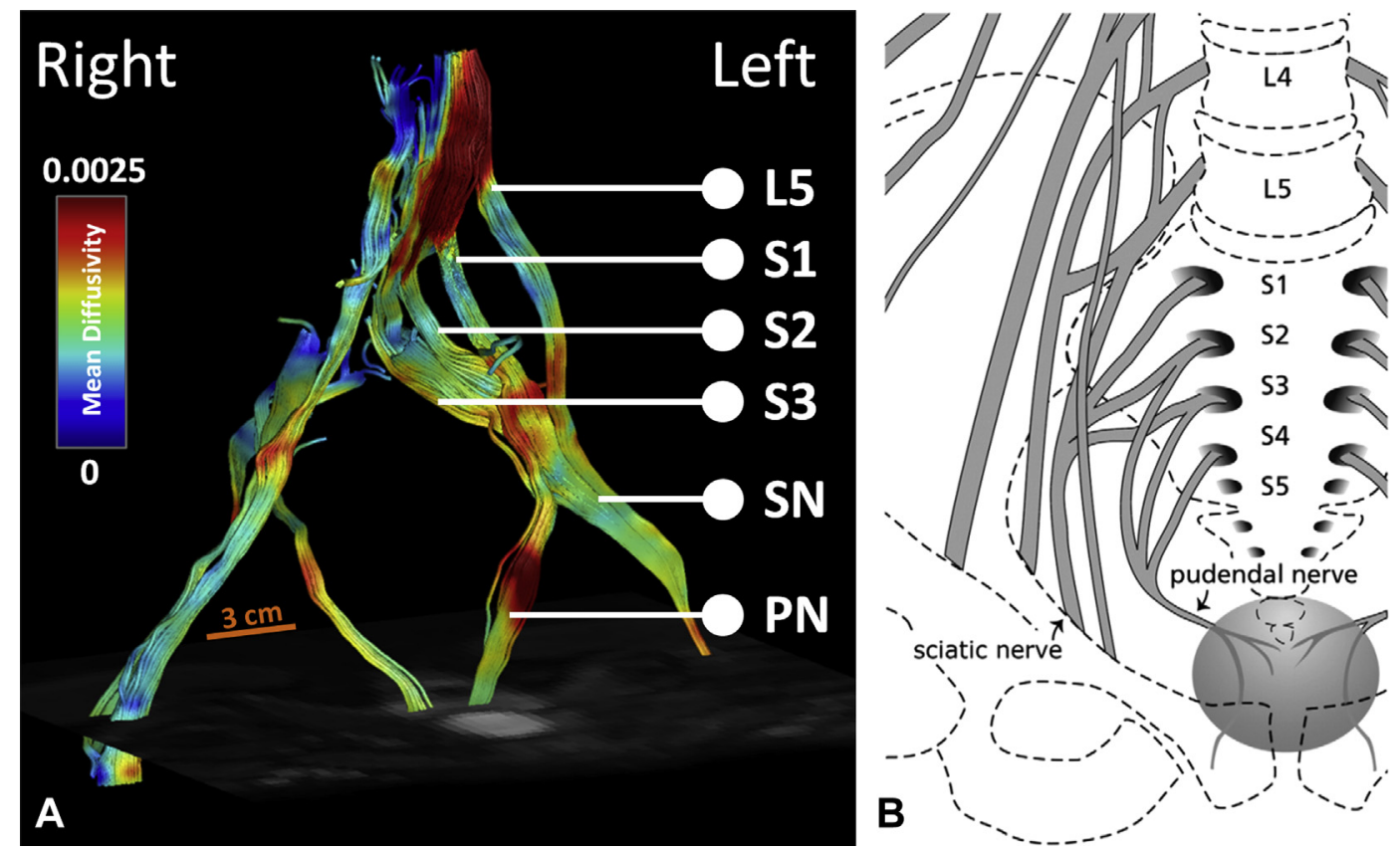

Figure 5. Patient with SB and myelomeningocele at L1-L4 lumbar level. $A$, anterior view. Note pudendal nerve $(P N)$ and sciatic nerve $(S N)$. $B$, overview of lumbosacral nerves. Anatomy corresponds to sacral branches and pudendal nerve $(A)$. 
associated with detrusor-sphincter dyssynergia compared to higher lesions. DTI could have an important role in diagnosis and therapy for future neuroanastomotic procedures in these patients.

\section{Limitations}

Data were corrected for subject motion and geometric distortions but may still have been misaligned due to the nonlinear behavior of these artifacts. ${ }^{5,9}$ In addition, due to the relatively large voxel size $(3 \times 3 \times 3 \mathrm{~mm})$ partial volume effects influenced the diffusion parameters and smaller nerve bundles, especially at S3 since those nerves are smaller in diameter than the other nerves. ${ }^{23,26}$ The control group consisted of healthy adults. Although diffusion properties may change with age, as in brain white matter fiber bundles, ${ }^{27}$ we expect that differences in $\mathrm{AD}$ and $\mathrm{RD}$ would be relatively low compared to differences in patients with SB vs healthy adults.

\section{Future Study}

To determine the extent to which diffusion parameters represent nerve functionality in patients with SB other patient groups with neurological disorders can be investigated. In newborns with SB DTI can be performed before and after closing the spinal cord as a comparison tool to localize potential nerve damage. By improving DTI resolution we believe that in the future it could be possible to visualize small nerves in these infants. Other pathological conditions for which DTI can be used to visualize the peripheral nerves include multiple sclerosis ${ }^{28}$ and paraplegia. Finally, DTI could potentially be applied to locate nerves for sacral nerve stimulation, neurostimulator implants and neuroanastomosis procedures ${ }^{29}$ in the sacral plexus. Combining electromyogram information with DTI tractography and diffusion parameters may improve our understanding of how to interpret diffusion parameters of peripheral nerves.

At this time DTI cannot replace conventional anatomical imaging modalities, ie 2D T1-weighted and T2-weighted sequences or high resolution 3D-TSE protocols. However, it can serve as a complementary tool to better detect and understand neurological problems in patients with SB. Further adjustments of the registration process by fusing anatomical and DTI information may improve analysis of the nerves at each level. Large-scale studies of patients with different types of SB are needed to further optimize the technique and better determine the exact role of DTI in the diagnosis and followup. We expect that abnormal diffusion parameters may indicate affected or dysfunctional nerves.

\section{CONCLUSIONS}

To our knowledge this 3 Tesla MRI study shows for the first time the asymmetry and disorganization of the sacral plexus in 10 patients with SB using DTI and FT. These abnormalities indicate that the sacral plexus of such patients differs from that of healthy controls. The observed difference in diffusion values shows that these methods can be used to identify nerve abnormalities. Combining 3D-TSE, DTI and FT, and correlating diffusion parameters with the neurological problems of patients with SB are expected to provide a valuable contribution to better analysis and diagnosis of these patients in the future.

\section{ACKNOWLEDGMENTS}

Niels Blanken, Department of Radiology, assisted with acquiring MRI data.

\section{REFERENCES}

1. de Jong TPVM, Chrzan R, Klijn AJ et al: Treatment of the neurogenic bladder in spina bifida. Pediatr Nephrol 2008; 23: 889

2. Dik P, Klijn AJ, van Gool JD et al: Early start to therapy preserves kidney function in spina bifida patients. Eur Urol 2006; 49: 908.

3. Özek MM, Cinalli G and Maixner WJ: Spina Bifida: Management and Outcome. New York: Springer 2008

4. de Groat WC: A neurologic basis for the overactive bladder. Urology 1997; 50: 36

5. van der Jagt PKN, Dik P, Froeling $M$ et al: Architectural configuration and microstructural properties of the sacral plexus: a diffusion tensor
$\mathrm{MRI}$ and fiber tractography study. Neuroimage 2012; 62: 1792

6. Matejcik V: Anatomical variations of lumbosacral plexus. Surg Radiol Anat 2010; 32: 409.

7. Basser PJ, Mattiello J and LeBihan D: MR diffusion tensor spectroscopy and imaging. Biophys J 1994; 66: 259.

8. Jones DK and Leemans A: Diffusion tensor imaging. In: Magnetic Resonance Neuroimaging. Edited by M Modo and JWM Bulte. New York: Humana Press 2011; pp 127-144.

9. Tournier JD, Mori $S$ and Leemans A: Diffusion tensor imaging and beyond. Magn Reson Med 2011; 65: 1532
10. Beaulieu C: The basis of anisotropic water diffusion in the nervous system-a technical review. NMR Biomed 2002; 15: 435.

11. Mori $S$ and van Zijl PCM: Fiber tracking: principles and strategies-a technical review. NMR Biomed 2002; 15: 468.

12. Hiltunen J, Suortti T, Arvela $S$ et al: Diffusion tensor imaging and tractography of distal peripheral nerves at 3 T. Clin Neurophysiol 2005; 116: 2315 .

13. Vargas MI, Viallon M, Nguyen D et al: Diffusion tensor imaging (DTI) and tractography of the brachial plexus: feasibility and initial experience in neoplastic conditions. Neuroradiology 2010; 52: 237. 
14. Wakana $\mathrm{S}$, Jiang $\mathrm{H}$, Nagae-Poetscher $\mathrm{LM}$ et al: Fiber tract-based atlas of human white matter anatomy. Radiology 2004; 230: 77.

15. Reijmer YD, Leemans $A$, Caeyenberghs $K$ et al: Disruption of cerebral networks and cognitive impairment in Alzheimer disease. Neurology 2013; 80: 1370.

16. Reijmer $Y D$, Brundel $M$, de Bresser $J$ et al: Microstructural white matter abnormalities and cognitive functioning in type 2 diabetes: a diffusion tensor imaging study. Diabetes Care 2013; 36: 137.

17. Takagi $T$, Nakamura $M$, Yamada $M$ et al: Visualization of peripheral nerve degeneration and regeneration: monitoring with diffusion tensor tractography. Neuroimage 2009; 44: 884.

18. Leemans A, Jeurissen B, Sijbers $J$ et al: ExploreDTI: a graphical toolbox for processing, analyzing, and visualizing diffusion MR data. Proc Intl Soc Mag Reson Med 2009; 17: 3536.
19. Leemans A and Jones DK: The B-matrix must be rotated when correcting for subject motion in DTI data. Magn Reson Med 2009; 61: 1336.

20. Veraart J, Sijbers J, Sunaert S et al: Weighted linear least squares estimation of diffusion MRI parameters: Strengths, limitations, and pitfalls. Neuroimage 2013; 81: 335.

21. Basser PJ, Pajevic S, Pierpaoli $C$ et al: In vivo fiber tractography using DT-MRI data. Magn Reson Med 2000; 44: 625.

22. Deprez $S$, Billiet $T$, Sunaert $S$ et al: Diffusion tensor MRI of chemotherapy-induced cognitive impairment in non-CNS cancer patients: a review. Brain Imaging Behav 2013; 7: 409.

23. Vos SB, Jones DK, Viergever MA et al: Partial volume effect as a hidden covariate in DTI analyses. Neuroimage 2011; 55: 1566.

24. Tournier JD, Calamante F, King MD et al: Limitations and requirements of diffusion tensor fiber tracking: an assessment using simulations. Magn Reson Med 2002; 47: 701.
25. Boemers TM, Van Gool JD, De Jong TPVM et al: Urodynamic evaluation of children with the caudal regression syndrome (caudal dysplasia sequence). J Urol 1994; 151: 1038.

26. Hogan 0: Size of human lower thoracic and lumbosacral nerve roots. Anesthesiology 1996; 85: 37.

27. Bennett IJ, Madden DJ, Vaidya CJ et al: Age-related differences in multiple measures of white matter integrity: A diffusion tensor imaging study of healthy aging. Hum Brain Mapp 2010; 31: 378.

28. Roosendaal SD, Geurts JJG, Vrenken $\mathrm{H}$ et al: Regional DTI differences in multiple sclerosis patients. Neuroimage 2009; 44: 1397.

29. Rasmussen MM, Clemmensen D, Rawashdeh YF et al: Surgical reinnervation with nerve anastomosis technique for neurogenic bladder and bowel dysfunction. Ugeskr Laeger 2011; 173: 2412.

\section{EDITORIAL COMMENT}

This is a preliminary report of a technology that would potentially have clinical application. It certainly might be advantageous to have a map of the neuroanatomy before surgical dissection. The technology would also seem to have application in helping define and predict neurological defects resulting from trauma and congenital malformation.
The study is preliminary but the technology presented seems to hold a glimmer of potential.

Michael Mitchell

Division of Pediatric Urology Children's Hospital of Wisconsin Milwaukee, Wisconsin 